\section{ESTIGMATIZACIÓN TERRITORIAL EN ACCIÓN ${ }^{1}$}

Loïc Wacquant ${ }^{2}$, Tom Slater ${ }^{3}$, Virgilio Borges Pereira $^{4}$

\section{Resumen}

Este breve artículo presenta el número temático ofrecido por la revista Environment E Planning A, la cual se basa en el marco analítico elaborado por Wacquant en su obra Urban Outcasts (2008) y en las actividades del Leverhulme Network on Advanced Urban Marginality con el objetivo sintetizar y estimular las investigaciones sobre el vínculo triangular entre el espacio simbólico, el espacio social y el espacio físico en el extremo inferior del espectro urbano. El concepto estigmatización territorial une el modelo elaborado por Goffman sobre el manejo de la "identidad deteriorada" con la teoría del "poder simbólico" desarrollada por Bourdieu para así capturar cómo un lugar estigmatizado puede afectar a residentes de barrios menospreciados, a sus vecinos, operadores comerciales, burocracias ciudadanas al nivel de impacto local, especialistas en producción cultural (periodistas, académicos y políticos), funcionarios públicos y políticas públicas. Este estigma espacial es un fenómeno nuevo y distintivo que se cristalizó a fines del siglo pasado junto con la disolución de los barrios de relegación

\section{TERRITORIAL STIGMATIZATION IN ACTION'}

\author{
Loïc Wacquant ${ }^{2}$, Tom Slater ${ }^{3}$ and Virgílio Borges \\ Pereira ${ }^{4}$
}

\section{Abstract}

This short article presents the thematic issue of the Environment \& Planning A journal, which builds on the analytic framework elaborated by Wacquant in Urban Outcasts (2008) and on the activities of the Leverhulme Network on Advanced Urban Marginality to synthesize and stimulate inquiries into the triadic nexus of symbolic space, social space, and physical space at the lower end of the urban spectrum. The concept of territorial stigmatization weds Goffman's model of the management of "spoiled identity" with Bourdieu's theory of "symbolic power" to capture how the blemish of place impacts the residents of disparaged districts, the surrounding denizens and commercial operators, street-level public bureaucracies, specialists in cultural production (such as journalists, scholars, and politicians), and state officials and policies. Spatial taint is a novel and distinctive phenomenon that crystallized at century's end along with the dissolution of the neighborhoods 
característicos de la etapa fordista-keynesiana del capitalismo industrial. Dicho fenómeno, equiparado a la desintegración social, difiere de la topografía tradicional de desprestigio de la ciudad industrial ya que se ha convertido en un evento autónomo, nacional, democrático y racialmente discriminatorio derivado de la acentuación selectiva, lo que suscita un rechazo que suele conducir a medidas sancionatorias. Las estrategias socio-simbólicas elaboradas por los residentes de barrios degradados para hacer frente a la denigración espacial cubren un amplio abanico que va desde la sumisión hasta la resistencia y su adopción depende de la posición y trayectoria dentro del espacio físico y social. La estigmatización territorial no es una condición estática o un proceso neutral, sino una forma significativa y perjudicial de acción mediante la representación colectiva centrada en un lugar determinado. Al indagar el modo de operación de este fenómeno en distintos escenarios urbanos y formaciones políticas, los colaboradores del número ofrecido por Environment and Planning A nos ayudan a progresar en nuestra comprensión sobre el papel de las estructuras simbólicas en la producción de desigualdad y marginalidad en la ciudad. También se sugiere la necesidad de diseñar políticas públicas enfocadas no sólo en la reducción de carencias materiales, sino también en la disminución de la presión ejercida por la dominación simbólica en la metrópolis.

PALABRAS CLAVE: ESTIGMATIZACIÓN TERRITORIAL, MARGINALIDAD AVANZADA, DESIGUALDAD URBANA， IDENTIDAD DETERIORADA， PODER SIMBÓLICO, ESTRATEGIAS SOCIALES, GOFFMAN, BOURDIEU of relegation emblematic of the Fordist-Keynesian phase of industrial capitalism. It differs from the traditional topography of disrepute in the industrial city in that it has become autonomized, nationalized and democratized, equated with social disintegration, racialized through selective accentuation, and it elicits revulsion often leading to punitive corrective measures. The sociosymbolic strategies fashioned by the residents of defamed quarters to cope with spatial denigration span a panoply ranging from submission to defiance, and their adoption depends on position and trajectory in social and physical space. Territorial stigmatization is not a static condition or a neutral process, but a consequential and injurious form of action through collective representation fastened on place. By probing how it operates in different urban settings and political formations, the contributors to the Environment and Planning A's issue advance our empirical understanding of the role of symbolic structures in the production of inequality and marginality in the city. They also suggest the need for public policies designed to reduce, not only the burden of material deprivation, but also the press of symbolic domination in the metropolis.

KEYWORDS: TERRITORIAL STIGMATIZATION, ADVANCED MARGINALITY, URBAN INEOUALITY, SPOILED IDENTITY, SYMBOLIC POWER, SOCIAL STRATEGIES, GOFFMAN, BOURDIEU 
1 El presente artículo se enmarca dentro del número especial de la revista internacional Environment and Planning A (vol. 46, no . 6, junio de 2014) dedicado a la "Estigmatización territorial". Esta contribución ofrece el marco analítico para las siete investigaciones recopiladas en dicha entrega. Revista INVI se complace en publicar la versión en español de este artículo para estimular el pensamiento de sus lectores sobre el papel de las estructuras simbólicas y la denigración espacial en los barrios de escasos recursos de toda América Latina. Asimismo, se aprovecha esta oportunidad para otorgar continuidad a las recientes visitas de Loïc Wacquant y Tom Slater a la Facultad de Arquitectura y Urbanismo de la Universidad de Chile en octubre de 2013 y abril de 2014, respectivamente.

2 Profesor de Sociología de la Universidad de California, Berkeley, e investigador en el Centro Europeo de Sociología y Ciencias Políticas de París. Becario en la Fundación MacArthury galardonado en 2008 con el Premio Lewis Coser de la Asociación Americana de Sociología, sus investigaciones se extienden a través de temas como relegación urbana, dominación étnico-racial, el Estado penal, realización, teoría social y la política de la razón. Sus libros han sido traducidos en más de 20 idiomas e incluyen la trilogía Urban Outcasts: A Comparative Sociology of Advanced Marginality (2008), Punishing the Poor: The Neoliberal Government of Social Insecurity (2009) y Deadly Symbiosis: Race and the Rise of the Penal State (2014), a los que se suma The Two Faces of the Ghetto (2014). Para mayor información, visite http://loicwacquant.net/

3 Profesor Adjunto de la cátedra de Geografía Urbana en la Universidad de Edimburgo. Sus intereses están orientados hacia la forma en que los nexos entre el mercado y las políticas estatales producen y refuerzan las desigualdades sociales en la ciudad. $\mathrm{Ha}$ escrito numerosos artículos sobre gentrificación (en especial el libro Gentrification [2008], del cual es coautor), los efectos de los barrios, estigmatización territorial y teoría urbana. Su investigación actual está enfocada en cómo los centros de estudios legitiman el constante asalto al Estado de bienestar británico. También se desempeña como coordinador en el Leverhulme Trust International Network on Advanced Marginality. Para mayor información, visite http://www.geos.ed.ac.uk/homes/tslater

4 Profesor Asociado de la cátedra de Sociología en la Universidad de Porto, donde realiza clases en la Facultad de Humanidades y de Arquitectura. Su trabajo moviliza las teorías y métodos de Bourdieu para explorar temas como desigualdad urbana, políticas de vivienda y las transformaciones históricas en los ámbitos de la clase y la cultura tanto en la ciudad como en el campo. Sus publicaciones han sido editadas en portugués, español, francés e inglés, donde se incluye Classes e culturas de classe das familias Portuenses (2005), Pierre Bourdieu, A teoría da prática e a constitução da sociologia em Portugal (del cual es coautor junto con J.M. Pinto, 2007), y Ao Cair do Pano: sobre a formação do quotidiano (ed., 2012). Para mayor información, visite http://www.isociologia.pt/investigacao.aspx?id=126
1 The present article is the introduction to the special issue of the international journal Environment and Planning A (vol. 46, nº. 6 , June 2014) on "Territorial Stigmatization». It provides an analytic frame for the seven articles gathered in that issue. Revista INVI is pleased to publish it in Spanish translation to stimulate its readers to think of the role of symbolic structures and spatial denigration in poor neighborhoods across the Latin American continent. Besides, this gives continuity to the recent visits of Loïc Wacquant and Tom Slater to the School of Architecture and Urban Planning of the University of Chile, on October 2013 and April 2014, respectively.

2 Professor of Sociology at the University of California, Berkeley, and Researcher at the Centre Européen de Sociologie et de Science Politique, Paris. A MacArthur Foundation Fellow and recipient of the 2008 Lewis Coser Award of the American Sociological Association, his research spans urban relegation, ethnoracial domination, the penal state, embodiment, and social theory and the politics of reason. His books have been translated in some 20 languages and include the trilogy Urban Outcasts: A Comparative Sociology of Advanced Marginality (2008), Punishing the Poor: The Neoliberal Government of Social Insecurity (2009), and Deadly Symbiosis: Race and the Rise of the Penal State (2014), as well as The Two Faces of the Ghetto (2014). For more information, see http://loicwacquant.net/

3 Reader in Urban Geography at the University of Edinburgh. He is interested in how the nexus of market and state policies produces and reinforces social inequalities in the city. He has written widely on gentrification (notably the co-authored book, Gentrification, 2008), neighbourhood effects, territorial stigmatization, and urban theory. His current research deals with how conservative think tanks legitimize the ongoing assault on the British welfare state. He is co-ordinator of the Leverhulme Trust International Network project on advanced marginality. For more information, see http://www.geos.ed.ac.uk/homes/tslater.

4 Associate Professor of Sociology at the University of Porto, where he teaches in the Faculties of Humanities and of Architecture. His work mobilizes the theories and methods of Bourdieu to probe urban inequality, housing policy, and historical transformations of class and culture in the city and the countryside. His publications have appeared in Portuguese, Spanish, French and English and include Classes e culturas de classe das famílias Portuenses (2005), Pierre Bourdieu, a teoria da prática e a construção da sociologia em Portugal (co-edited with J.M. Pinto, 2007), and Ao Cair do Pano: sobre a formação do quotidiano (ed., 2012). For more information, see http://www.isociologia.pt/ investigacao.aspx?id=126 
Durante las sesiones de la Asociación Internacional de Sociología que se llevaron a cabo en Barcelona en 2008, un grupo de jóvenes investigadores interesados en la estructura cambiante, las dinámicas y la experiencia de la marginalidad a nivel internacional se reunió en torno a un proyecto basado en la evaluación de las pruebas empíricas y en la extensión del marco analítico propuesto por Loïc Wacquant ${ }^{5}$ en Urban Outcasts respecto a las nuevas ciudades, países y ámbitos ${ }^{6}$. Estos investigadores compartían la opinión, fundamental del libro, de que la relegación en la ciudad no se da con los mismos patrones a pesar de la cada vez mayor presencia de fuerzas transnacionales y discursos normalizadores. Sin embargo, este fenómeno obedece a mecanismos similares y muestra una serie de características sociales y espaciales relacionadas que merecen ser estudiadas. El deseo de este grupo era evitar el error común de aislar artificialmente a

\footnotetext{
Wacquant, 2008.

Los cinco principios analíticos del libro propuestos para el estudio comparativo de la marginalidad urbana a nivel espaciotiempo (Wacquant, 2008, págs. 7-12) son: (1) establecer una clara demarcación entre conceptos populares y analíticos; (2) contextualizar las formas urbanas en el largo plazo; (3) utilizar la etnografía como un instrumento de ruptura epistémica y construcción teórica; (4) establecer diferencias entre vecindarios y marginación por medio de su condición, ubicación y función dentro del contexto metropolitano; y (5) especificar el grado y tipo de participación del Estado en zonas desfavorecidas. También se formuló un principio adicional: (6) ubicar el territorio de relegación dentro del amplio campo de formas de aislamiento socio-espaciales que dominan en las ciudades y en las sociedades (Wacquant, 2010).
}

la gente de escasos recursos en espacios sociales y buscaron, en cambio, vincular las transformaciones que corrompen los barrios bajos de la metrópolis con las estrategias y luchas que atraviesan los distintos círculos de poder ${ }^{7}$. Este colectivo de investigadores aneblaba un diálogo teórico guiado a nivel internacional que los ayudara a evitar quedar atados en los reducidos parámetros de sus debates locales y a protegerse de la oculta subordinación del ámbito académico a las agendas políticas ${ }^{8}$. Al poco tiempo se formó una red internacional (coordinada a través del sitio web advancedurbanmarginality.com) encargada de reunir a estudiantes de metrópolis de diferentes países y disciplinas quienes adoptaron un enfoque basado en la exploración en terreno (en solitario o en combinación con métodos históricos y estadísticos) a tono con la textura de la vida social del día a día. Este grupo se propuso indagar la interacción dinámica entre 
patrones espaciales, divisiones simbólicas y acción social y subrayar el rol multidimensional del Estado y los diferentes detentadores del capital económico y cultural en la producción, distribución y representación de distintos tipos de problemas y territorios en el contexto de la ciudad.

Con el apoyo del Leverhulme Trust y de las casas de estudios de sus principales miembros, esta red organizó una serie de actividades entre las cuales destacan un taller realizado en la Universidad de Edimburgo en 2010, un simposio internacional sobre "Estigmatización territorial" celebrado en la Universidad de Porto en junio de 2011 y una conferencia de dos días sobre "Marginalidad urbana y Estado" que tuvo lugar en el Collège de France, París, en el verano de 2012, a la cual asistieron académicos de una decena de países. El número temático ofrecido por la revista Environment \& Planning A sobre la lógica detrás de la "Estigmatización social" se basa en estos eventos y es el resultado del trabajo colectivo de una red de asociados que abarca disciplinas como la sociología, geografía, antropología, ciencias políticas, historia, criminología, arquitectura y urbanismo y trabajo social. Este es un esfuerzo destinado a sintetizar y estimular las investigaciones sobre el vínculo triangular entre espacio simbólico (divisiones mentales estipuladas en categorías), espacio social (distribución eficiente

ARTíCULO: Estigmatización territorial en acción / Loïc Wacquant, Tom Slater, Virgilio Borges Pereira de recursos entre estas categorías) y espacio físico en el extremo inferior del espectro urbano.

Wacquant ${ }^{9}$ ha relatado cómo se encontró con esta complicada realidad del estigma espacial durante el curso de una de sus investigaciones en terreno a nivel transatlántico a principios de la década de 1990, la cual estaba destinada a comparar la experiencia común de vivir en un degradado hiperguetto al interior de la ciudad de Chicago y en las deterioradas urbanizaciones ubicadas en las afueras de París. En ambos lugares, sus residentes expresaron la fuerte y categórica opinión de vecinos, funcionarios estatales y medios de comunicación comerciales respecto a la apreciación negativa de sus barrios como nidos de veneno social, viciosos y violentos. En este sentido, estos habitantes extendieron el estigma de vivir en un área juzgada como purgatorio socio-moral hacia otros individuos, validando y esparciendo así los efectos de este problema. Asimismo, este grupo de personas concibió estrategias para evitar todo tipo de asociación con este tipo de lugares, entre las que se incluyen la no revelación de sus direcciones, abstenerse de invitar a terceros a sus hogares, refugiarse dentro del núcleo familiar, restringir su participación en grupos locales y migrar de su lugar de residencia ante la primera oportunidad que se presente. Lo curioso de este tema es que el estigma que recae sobre

9 Wacquant, 2009, p. 115-117 
estos lugares logró afectar las actitudes y acciones de empleados públicos de alto nivel a cargo de la recientemente instituida política urbana de Francia. Por ejemplo, estos funcionarios consideraron que operar en uno de estos oficialmente designados "barrios sensibles" dejaba una marca negra y constituía un impedimento en sus aspiraciones de desarrollo profesional. En el caso de Chicago, el estigma espacial que se ancló sobre los derruidos guetos fue fruto de un trato diario que sembró el desprecio y temor generalizado hacia sus habitantes, convirtiéndose en la base de la discusión periodística y política que tildaba al Cinturón Negro como la "cruz de la sociedad norteamericana"10 y transformándose en un tema dóxico explotado por una floreciente vertiente académica a cargo de investigar el supuesto auge y diseminación de una alarmante "subclase". 11

10 Chicago Tribune, 1986

11 Para acceder al texto emblemático que reúne los constituyentes clave de este negativo mito académico y su respectiva actualización histórica, consultar Jencks y Peterson (1991) y Katz (2013) respectivamente. La dimensión espacial de esta categoría se ve reforzada por la invención del concepto de "áreas de subclase", el cual es un distrito donde se concentran diversas patologías.

224 revista invi № 82 / Noviembre 2014 / Volumen № 29: 219-240
Wacquant se alineó con la visión de Ervin Goffman ${ }^{12}$, la cual considera que los estigmas son "diferenciaciones basadas en el desprestigio" que surgen de la mirada común del resto. En este sentido, existe una directa interacción con la teoría de Pierre Bourdieu ${ }^{13}$ sobre el poder simbólico, la cual es vista como una "nominación performativa" ejercida por una autoridad capaz de representar y forjar el concepto de estigmatización territorial. Esta noción capturó la propiedad simbólica más destacada que, junto a la fragmentación del trabajo asalariado en el ámbito material, fue configurando una "marginalidad avanzada" en la metrópolis dual de fines del siglo pasado. ${ }^{14} 15$ De acuerdo a la visión de Goffman, estos estigmas están distribuidos en tres clases amplias basadas en la "abominación del cuerpo", los "defectos del carácter individual" y la afiliación "tribal", la cual es "transmitida por medio del linaje"; a ellos se

12 Goffman, 1963

13 Bourdieu, 1991.

14 Wacquant, 2007, 2008 y 2010

15 "Marginalidad avanzada" es un régimen de pobreza ascendente en las ciudades postindustriales de la sociedad avanzada en e contexto del resurgimiento de la desigualdad de clases, el retroceso del Estado de bienestar, la expansión del Estado penal y la polarización espacial. Esta mancha territorial es la tercera de las seis propuestas que caracterizan este problema (Wacquant, 2008, capítulo 8): (1) la creciente heterogeneidad interna y la marginación laboral, (2) la desconexión funcional entre los barrios y las tendencias macroeconómicas; (3) fijación y estigmatización territorial; (4) aislamiento y disolución espacial; (5) la pérdida de un entorno viable; $y$ (6) la fragmentación de poblaciones marginadas, las cuales son pulverizadas más allá de los ámbitos comprendidos por colectivos establecidos (tales como sindicatos, organizaciones comunales y partidos políticos de izquierda). 
suma del aporte de Wacquant ${ }^{16}$ referente a la identificación del espacio como un anclaje distintivo de deslegitimación social. Asimismo, la proposición hecha por Bourdieu sobre el poder simbólico y su participación en la "formación y desintegración de grupos" mediante la reducción del espacio social en formas que (des)movilizan a sus miembros putativos se ve expandida por la contribución de Wacquant, quien incorpora la mediación crucial del lugar como un contenedor de material, encrucijadas sociales e imágenes mentales cargadas de profundos valores emocionales a través de los cuales emergen grupos que se abren paso por medio de reclamaciones sobre el espacio ya construido. Estas dos perspectivas teóricas no sólo son compatibles sino que se complementan entre si $^{17}$ : Bourdieu analiza

16 Wacquant, 2014a.

17 La opinión común respecto al hecho de que Bourdieu y Goffman sean teóricos sociales discordantes (donde el trabajo de Jensen y Peterson [2012] se convierte en un problema para el concepto de mancha espacial elaborado por Wacquant) es el resultado de la confluencia de interpretaciones erróneas en torno a sus respectivas posturas: mientras que Bourdieu es frecuentemente interpretado de manera incorrecta como un "estructuralista" mecánico que no puede situar la acción creativa a pequeña escala (a pesar de la fluidez de su díada conceptual sobre habitus y espacio social), Goffman es comúnmente encasillado como un "interaccionista simbólico" en la línea de Blumer, cuando en realidad es un seguidor acérrimo de la escuela de Durkheim que indaga la morfología social y las representaciones colectivas propias del "orden de la interacción". No es de extrañarse que Bourdieu fuese un seguidor, admirador intelectual y amigo personal de Goffman, cuyas obras se encargó de traducir al francés en una serie de libros publicados por la editorial vanguardista Minuit. el tramo superior de este asunto al explorar las representaciones y repercusiones de autoridades simbólicas como el Estado, la ciencia, la iglesia, el derecho y el periodismo en el funcionamiento de las instituciones, las prácticas sociales y nosotros mismos; mientras que Goffman, por su parte, analiza el tramo inferior del tema en cuestión mediante el seguimiento de los efectos de procedimientos destinados a otorgar sentido a las cosas y de las técnicas de "manejo de la identidad dañada" en el contexto de encuentros e incorporaciones dentro de diversas organizaciones. De esta manera, estas dos visiones pueden relacionarse para ayudarnos a comprender mejor la forma en que estas representaciones nocivas se reproducen, diseminan y utilizan tanto en el ámbito del poder, de la mano 
de agencias burocráticas y comerciales, como en la vida diaria, afectando la identidad, estrategias y estructuras a nivel social.

Cabe destacar que, a modo de preludio del número temático ofrecido por Environment and Planning A, la tesis sobre estigmatización barrial es descrita como una fenómeno nuevo y distintivo que se cristalizó a fines del siglo pasado junto con la repentina descomposición o disolución gradual de los barrios de relegación característicos de la etapa fordista-keynesiana del capitalismo industrial: a saber, el gueto negro de los Estados Unidos, los barrios tradicionales habitados por la clase trabajadora en la deteriorada ciudad central o en la periferia metropolitana de Europa y los estabilizados barrios desfavorecidos de toda América Latina ${ }^{18}$. Este reclamo no implica que la topografía de desprestigio sea un fenómeno nuevo derivado de la metrópolis postindustrial. De hecho, es bien sabido que "una parte maldita" de la sociedad urbana ha sido confinada en

18 Wacquant, 2014b. distritos especiales, bas-fonds, barrios marginales, colonias superpobladas y submundos desde el siglo XIX como resultado de la confluencia del desarrollo urbano, la industrialización y los miedos y fantasías de las clases superiores en torno a las "masas pululantes" que se concentraban en la ciudad. La clásica representación del barrio irlandés de Manchester descrita por Friedrich Engels ([1845] 1993) en la obra The Condition of the Working Class in England y el retrato detallado de Covent Garden ofrecido por Robert Mayhew ([1851] 2012) en London Labour and the London Poor son suficientes para atestiguar esta situación. Hacia finales del siglo XIX, los barrios de Montfaucon en París, Five Points y Bowery en Nueva York, Saint Giles y el East End en Londres -así como La Boca en Buenos Aires y Casbah en Argel- fueron tristemente famosos por ser antros deplorables de "indigencia, delincuencia y disipación". ${ }^{19}$ Sin embargo, el estigma que recae

19 Kalifa $(2012$, p. 16) ofrece un detallado panorama histórico sobre el nacimiento, evolución y disipación de los bas-fonds como elemento principal del imaginario occidental que se consolidó a mediados del siglo XIX, el cual tiene sus raíces en el surgimiento del concepto de "pobres no merecedores de asistencia" durante el siglo XIII y la estigmatización de los vagabundos y gitanos provenientes de la región de Bohemia que tuvo lugar en el siglo $\mathrm{XVI}$. Este autor sostiene que este imaginario se expandió durante la primera mitad del siglo XX como resultado del cambio en las representaciones colectivas (socialización de la pobreza, el establecimiento del desempleo como categoría oficial y los delincuentes comenzaron a ser vistos como parte de un entorno dife rente) y desapareció durante la segunda mitad del siglo pasado a raíz de las mejoras en el estado económico y situación habitacional de la clase trabajadora. 
sobre los barrios desfavorecidos de hoy difiere de la marca espacial de antaño en al menos cinco aspectos.

En primer lugar, si bien es un concepto que ha alcanzado cierta autonomía, el estigma territorial guarda una estrecha relación con la pobreza, la subordinación étnica (donde se incluyen "minorías" nacionales y regionales, ya sean reconocidas o no, y grupos de inmigrantes extranjeros pertenecientes a las clases bajas), viviendas degradadas, moralidades impuestas y delincuencia callejera. Esta situación ha llegado a tal punto que una nueva etiqueta genérica, creada para referirse a este tipo de barrios como rasgaduras y amenazas que afectan al tejido de una nación, ha sido ampliamente aceptada en países desarrollados: algunos ejemplos son banlieue-ghetto en Francia, quartieri degradati en Italia, Problemquartier en Alemania, sink estates en el Reino Unido y krottenwijk en Holanda, entre muchos otros. Segundo, el estigma territorial se ha convertido en un fenómeno nacional y democrático: en cada país, un pequeño grupo de barrios se ha vuelto universalmente reconocido y atacado a nivel social y espacial por constituirse en refugios donde la indigencia y la decadencia son características que se generan y perpetúan por sí solas. Estos nombres -sinónimos de infiernos sociales- circulan tanto en discursos periodísticos, políticos y académicos como en conversaciones de todos los días. Esta imagen sulfurosa no sólo prevalece entre las élites sociales y culturales -como sus predecesores hace un siglo atrás- sino que entre toda la ciudadanía en general, incluyendo aquellos individuos que viven en estos lugares de rechazo y otros tantos que han sido desterrados de estos espacios. En este sentido, los habitantes de las remotas villas de Suecia tiemblan ante la simple mención de barrios como Rinke y, Tensta y Fittja, aunque ni siquiera hayan estado o probablemente nunca se aproximarán a estas tristemente célebres "áreas prohibidas" ubicadas en los alrededores de Estocolmo. ${ }^{20}$ Este tipo de perdición urbana ha sido incluso difundida a nivel internacional a través de películas, videos musicales y juegos de video, en lugar de las novelas y periódicos del siglo XIX. De esta manera, "el Bronx" se ha convertido en el denominador común para referirse a calderas de decadencia física, moral y social en ciudades de 
países tan lejanos como Argentina, Francia y Australia. $^{21} 22$

Tercero, mientras que los barrios estigmatizados derivados de la ciudad postindustrial son descritos como vórtices y vectores de desintegración social, en esencia disolutos e irremediablemente desorganizados, el "contra-mundo" de los bas-fonds del período clásico industrial era visto como "una contra-sociedad poderosa y jerarquizada", un "doble invertido, una falsificación y una caricaturización de la sociedad organizada" que la rodeaba. ${ }^{23}$ La denominación "gueto" se utiliza generalmente para dramatizar y denunciar dicha desintegración; esta etiqueta es incluso empleada por académicos, inconscientes del tenor sociológico de la marginación como mecanismo de integración estructural ciudadana de grupos étnicos estigmatizados. ${ }^{24}$ Este título nos dirige a una cuarta diferencia: la

21 Auyero, 1999; Wacquant, 2008; Birdsall-Jones, 2013.

22 Durante el último tiempo, el barrio de Rosengård, ubicado en Malmö, ha ganado una oscura notoriedad en gran parte de Europa como resultado de las peregrinaciones profesionales de la estrella de fútbol sueca Zlatan Ibrahimović, quien es siempre presentado en distintos medios locales como el "oriundo del gueto" de Rosengård. En sus entrevistas, así como en su autobiografía (Ibrahimović y Lagercrantz, 2014), Ibrahimović utiliza esta visión nociva ampliamente difundida para dramatizar su ascenso, rodear sus habilidades en un manto de misterio, disculparse por su conducta poco convencional y reafirmar su lealtad innata (suele citar la variante formal de la máxima afroamericana "puedes sacar a un hombre del gueto, pero no puedes sacar al gueto del hombre").

23 Kalifa, 2012, p. 61-66

24 Wacquant, 2011a. discriminación racial derivada de la acentuación selectiva o proyección ficticia. Las poblaciones de estos barrios degradados casi siempre son vistas desde una perspectiva más oscura y exótica que la ofrecida por su esfera demográfica. Sus diferencias culturales son exageradas y convertidas en divergencias, e incluso hostilidades, en relación a las normas dominantes a nivel nacional -donde la religión actúa como agente oculto de sedición-, mientras que su vulnerable posición social es minimizada o ignorada por completo. Todo incidente que involucre alguna anormalidad o violencia dentro o alrededor de estas áreas es habitualmente explotado con fines sensacionalistas y vinculado con la supuesta característica intrínseca de sus residentes, quienes son catalogados como marginados. En este sentido, todo vecindario que cuente con una pequeña y estable minoría de residentes inmigrantes o de 
raza negra, bajas tasas de delincuencia y viviendas básicas pero adecuadas puede ser convertido rápidamente en un gueto racial y hostil listo para explotar en caos en cualquier minuto, como es el caso de la imagen colectiva impuesta al barrio de St. Pauls en Bristol. ${ }^{25} 26$

25 Slater y Anderson, 2012.

26 Los constantes choques entre jóvenes locales y la policía son rápidamente elevados a la categoría de "disturbios raciales" sólo por el tono más oscuro de la piel de los alborotadores. La retórica marcial que hace referencia a la "guerra" es expuesta y mezclada con el vocabulario racial relacionado con el (post) colonialismo para generar una visión explosiva de un total e irresoluble conflicto urbano cuyas raíces se remontan siglos atrás hacia el cruel conflicto que enfrentó a la civilización cristiana e islámica. Estas revueltas son precisas para atraer la atención de los medios de comunicación y vender libros, como es el caso de Andrew Hussey y su absurda obra The French Intifada: The Long War Between France and its Arabs (2014: 12), donde es posible leer: "Los alborotadores en Gare du Nord o en los banlieues con frecuencia se describen a sí mismos como soldados luchando una 'larga guerra' contra Francia y Europa. En este sentido, ellos están combatiendo el concepto mismo de of 'civilización', el cual es percibido como una invención de Europa. La supuesta 'intifada francesa', la guerrilla contra la policía en los suburbios y en el centro de las ciudades francesas es sólo la última y más dramática forma de enfrentarse al enemigo. Esta guerra tuvo sus inicios en la cínica agresión a Egipto, ordenada por Napoleón a comienzos del siglo XVII, lo que gatilló el inició de la fascinación francesa por todo lo oriental" y que ahora emerge como la "cuarta guerra mundial" que tiene enfrentados a Francia contra Argelia, Túnez y Marruecos. En vez de ser motivo de una desconfiada hilaridad, este libro ha recibido críticas muy favorables en la prensa británica (el Sunday Times lo catalogó como una obra "fascinante, convincente y altamente recomendable") y seguramente gozará de muchos elogios en sectores académicos donde vociferaciones retóricas actúan como un sustituto barato para la investigación.
Por último, pero no menos importante, estos barrios estigmatizados y desfavorecidos de la ciudad postindustrial suscitan una gran cantidad de emociones negativas y reacciones severas correctivas impulsadas ya sea por miedo, repulsión y rechazo, lo que a su vez da paso al crecimiento 
y glorificación de la faceta penal del Estado como una forma de castigar este tipo de marginación urbana. ${ }^{27}$ Atrás quedó la fascinación ambivalente y la espeluznante atracción de las élites políticas y culturales por los sórdidos bas-fonds de la emergente ciudad industrial. En estos lugares las clases altas encontraban emociones y espacios misteriosos de voyerismo social, transgresión moral, fantasías sexuales e inspiración artística, como lo demuestra la creación simultánea del periodismo de "suburbio" y de tipo "encubierto", el cual se centraba en estos derruidos barrios de la metrópolis. ${ }^{28}$ Durante la década de 1880, el segmento alto del Londres victoriano repletaba buses para dirigirse, durante la medianoche, desde sus lujosos enclaves hacia los suburbios del este de la ciudad para observar de primera mano el destellante espectáculo del "pauperismo" y admirarse con las extravagantes

27 Wacquant, 2009b; también consultar Clear, 2007; Muchielli, 2008; Peck y Theodore, 2008; Beckett y Herbert, 2011.

28 Kalifa, 2012. visiones, sonidos y escenas ofrecidas por sus conciudadanos, quienes se encontraban sumidos en la indigencia. ${ }^{29}$ Hacia la década de 1980, ningún habitante adinerado de Chicago hubiese pensado, por no decir osado, en dirigirse y adentrarse en el Robert Taylor Homes, ubicado en el lado sur de la ciudad, menos aún durante la noche. Hoy en día, cada vez que personas de poder y eminencia visitan estos barrios se anuncian medidas para erradicar flagelos, restaurar el orden y castigar a los delincuentes, esto ocurre con mucha frecuencia dentro de un contexto marcial. Un ejemplo de esto es el degradado vecindario de Easterhouse, ubicado en Glasgow, el cual es constantemente visitado por miembros del partido conservador en sus esfuerzos para obtener apoyo por las retrógradas reformas de bienestar que se han implementado en toda Gran Bretaña. ${ }^{30} 31$

30 Slater, 2014

31 Al otro lado del Canal de la Mancha, la visita más recordada al tristemente famoso proyecto habitacional de Quatre Mille, ubicado en la comuna de La Courneuve, fue encabezada por Nicolás Sarkozy el 20 de junio de 2005. En esa ocasión, el entonces Ministro del Interior y futuro Presidente de Francia prometió, en respuesta a un incidente criminal, que utilizaría una Karcher (una manguera de alto poder) para "limpiar toda la basura" de aquel lugar. Esta visita fue seguida por la presentación de un cuerpo de policía de estilo militar, compuesta por más de 200 oficiales, desplegada especialmente para los medios de comunicación. A pesar de no llevar a cabo ningún arresto o decomiso de objetos saqueados, esta fuerza fue seguida de cerca por las cámaras de televisión y difundida durante la franja noticiosa de las ocho de la noche. 
La estigmatización territorial descrita en párrafos anteriores no es sólo un nuevo fenómeno urbano característico de la metrópolis postfordista, también es una forma significativa y profunda de expansión de acciones a través de representaciones mentales y objetales. ${ }^{32}$ Las contribuciones recopiladas en esta entrega tienen efectos sobre la forma de sentir, pensar y actuar de una gran cantidad de agentes involucrados a medida que se difunden y diseminan a través de las estructuras sociales y espaciales de la ciudad. Desde los niveles más bajos hasta las más altas esferas gubernamentales, la denigración espacial tiene efectos sobre:

(1) Los residentes de barrios degradados, puesto que se corroe el sentido del sí mismo, se tuercen sus relaciones sociales y se debilitan sus capacidades de acción colectiva, ya que emergen estrategias de afrontamiento que tienden a validar, amplificar y proliferar su condición de desprestigio en su nivel fundamental, incluso cuando algunos tratan de ignorar o resistir este estigma espacial;

(2) Los habitantes y operadores comerciales, como lo demuestran los patrones de evitación entre vecinos y la "discriminación respecto a la dirección de residencia" de parte de empleadores;

32 Bourdieu, 1991, págs. 220-221.
(3) El nivel y calidad de los servicios prestados por burocracias ciudadanas tales como bienestar, salud y protección policial (donde el despliegue insuficiente fuerzas de vigilancia o tácticas agresivas serían inaceptables en otros sectores de la ciudad);

(4) El trabajo de especialistas en producción simbólica, tales como periodistas, académicos, analistas de políticas y funcionarios políticos.

(5) Las creencias, visiones y decisiones de funcionarios públicos y la resultante gama de políticas públicas que, en combinación con el mercado y otro tipo de fuerzas, determinan y distribuyen la marginalidad y todas sus consecuencias.

Durante su presentación en la Universidad de Porto, Wacquant ${ }^{33}$ examinó su marco analítico y propuso establecer diferencias entre las estrategias sociales y simbólicas elaboradas por los habitantes de barrios estigmatizados según si ellos se rinden y reproducen, o desafían y eluden, este estigma espacial. Wacquant sugirió que la propensión a adoptar una u otra estrategia (y enlazarlas dentro de cadenas coherentes) depende de la posición y trayectoria dentro del espacio físico y social. Así, este tipo de elecciones variarán significativamente de acuerdo a distinciones como clase, edad y etapa

33 Wacquant, 2011b.

revista invi № 82 / Noviembre 2014 / Volumen Nº 29: 219-240 231 
dentro del ciclo de vida, régimen de tenencia de vivienda, antigüedad dentro del barrio y origen étnico (definido por la escuela de Weber como un reclamo válido de honor). (ver tabla 1).

Consideremos las siguientes dos situaciones ideales a modo de ejemplo. Ceteris paribus, la cual es una categoría donde un anciano propietario que tiene a muchos parientes viviendo en su mismo barrio -en una posición económicamente seguratiene más probabilidades de refugiarse dentro del ámbito familiar, apartándose y denigrando a sus vecinos (en línea con las representaciones dominantes reflejadas en las estrategias 4, 2 y 3), que emigrar fuera de su lugar de origen o reivindicar la identidad negativa atribuida a sus ocupantes (estrategias 5 y 8). Por otra parte, es más probable que el joven hijo de inquilinos inmigrantes que recientemente se establecieron en un área determinada acepte esta mancha territorial y practique un "alineamiento grupal"34 con sus pares adolescentes, celebrando e incluso alardeando sobre la maldad de su barrio o cité en un esfuerzo colectivo destinado a invertir este estigma; mientras que sus padres optarían por dejar ese lugar o desarrollar un frente de indiferencia (estrategias 5 y 8). Sin embargo, este individuo reconoce la nula funcionalidad de

34 Goffman, 1963, p. 112-114.

232 revista invi № 82 / Noviembre 2014 / Volumen № 29: 219-240 estos esfuerzos, altera su forma de vestir, modifica su discurso de auto presentación fuera del barrio de origen y miente sobre su dirección de residencia al momento de postular a algún puesto de trabajo (Truong, 2013). En este contexto, la aparentemente incongruente relación entre las estrategias 1 y 8 se ve estabilizada por la estricta separación de las escenas donde ocurre cada evento.

El número temático ofrecido por Environment and Planning A recopila siete estudios a fondo sobre la estigmatización territorial en ocho escenarios nacionales distribuidos en tres continentes (Francia, Portugal, Canadá, Australia, Escocia, Holanda, Turquía y Dinamarca). Por medio de diversas estrategias de investigación, estos artículos recorren dimensiones que van desde la vida cotidiana hasta las más altas esferas del Estado, validando, complicando y cuestionando el marco elaborado por Wacquant al llevarlo a nuevos terrenos geográficos, empíricos y analíticos. ${ }^{35}$ Colectivamente, estas investigaciones confirman la penetración, cargas existenciales e intrincadas reverberaciones asociadas a la estigmatización espacial y a las vidas de los residentes de distritos relegados; amplían y profundizan el repertorio de estrategias de enfrentamiento, resistencia y evasión desarrolladas por

35 Para obtener un panorama más amplio respecto a estudios recientes sobre la noción de estigmatización territorial a través de distintos temas, divisiones disciplinarias, sitios urbanos y fronteras nacionales, consultar la bibliografía preparada por Slater et al. (2013) disponible en advancedurbanmarginality.com.

ARTíCULO: Estigmatización territorial en acción / Loïc Wacquant, Tom Slater, Virgilio Borges Pereira 
este grupo de personas; y demuestran cómo el estigma que recae sobre un lugar se ve alimentado, utilizado y manipulado por intereses privados (tales como los medios de comunicación, empleados y empresas inmobiliarias) y funcionarios públicos (en el ámbito político y burocrático) con el fin de promover sus propias agendas, de manera que la desgracia espacial opera como un eje simbólico entre desigualdad y marginalidad en la metrópolis.

En su artículo The Cités Strike Back, Paul Kirkness ${ }^{36}$ recopila dos años de investigación en terreno en dos barrios degradados ubicados en Nîmes, al sur de Francia, para examinar detalladamente cómo sus habitantes reconocen de forma simultánea y hacen frente al estigma territorial. Este autor subraya las constantes prácticas de "emplazamiento", mediante las cuales los habitantes de un barrio determinado pretenden obtener una recuperación táctica del entorno espacio-vida, y la ejecución de "contra-guiones", donde habitantes intentan proyectar imágenes positivas de su barrio y de sí mismos. Sin embargo, su resistencia a la estigmatización territorial los mantiene profundamente inmersos en una nefasta red de simbolismo basado en la súplica. Esta perdurable "ambivalencia sociológica"37 es la que prevalece entre los habitantes de Bairro do Viso, uno de los asentamientos de escasos recursos más grandes y tristemente célebres de Portugal.

36 Kirkness, 2014.

37 Merton, 1976.
Pereira y Queiros ${ }^{38}$, en su colaboración titulada It's not a Bairro, Is It?, relacionan conceptos etnográficos, entrevistas semiestructuradas y una encuesta por muestreo a familias locales en el contexto de la historia habitacional de la ciudad de Porto para elaborar un mapa sobre divisiones sociales, formas de sociabilidad comunes y fronteras simbólicas que establecen patrones de interacción con el Estado. Estos autores descubren que los habitantes de este sector reaccionan ante la estigmatización territorial por medio de la limitación de sus intercambios sociales a actividades de subsistencia y aceptan, de modo nostálgico, que el área donde viven ya no es más un barrio. Estas respuestas estratégicas basadas en la "sociabilidad de subsistencia" y en la "evitación concentrada" afectan las dualidades salida-opinión y conformidad-rechazo y preparan el camino hacia un análisis más cuidadoso sobre el manejo de la estigmatización espacial en la vida cotidiana.

Martine August, en su artículo Challenging the Rhetoric of Stigmatization ${ }^{39}$, explota el tema del vínculo reacio y destaca los beneficios olvidados de la pobreza concentrada en el tristemente célebre barrio de Regent Park, ubicado en Toronto. Si bien este primer proyecto de viviendas sociales de Canadá ha estado rodeado de discursos descendentes sobre aislamiento, desorganización y peligro, este

38 Pereira y Queiros, 2014.

39 August, 2014. 
tipo de relatos ha sido últimamente mencionado para justificar el proceso de gentrificación impulsado por el Estado por medio de una remodelación "socialmente mixta". Sin embargo, a pesar del deterioro físico y la nefasta reputación de sus barrios, entrevistas realizadas a distintos vecinos revelan que existe una valoración respecto a sus espacios de residencia por el hecho de ser lugares que albergan amplias redes de amistad y apoyo recíproco, cercanía de equipamiento urbano y agencias locales que ofrecen servicios específicamente adaptados a sus necesidades. Este complejo habitacional se constituye como un "lugar' comunal sumergido en emociones compartidas y significados conjuntos sostenidos por prácticas e instituciones de mutualidad" en vez de convertirse en un "espacio' de indiferencia donde simplemente imperan la sobrevivencia y las disputas incesantes" ${ }^{\prime 40}$, de acuerdo a la visión política detrás de esta remodelación. Por otra parte, Arthurson, Darcy y Rogers ${ }^{41}$ nos llevan a Australia para indagar la recurrente desconexión entre imágenes dominantes y subordinadas de marginalidad urbana y sus respectivas consecuencias. Estos autores analizan cómo los inquilinos de viviendas sociales en Sídney y Adelaida perciben, reaccionan y reflexionan sobre el

40 Wacquant, 2008, pág. 241.

41 Arthurson, Darcy y Rogers, 2014. "estigma territorial televisado" difundido por la serie de ficción Housos. Esta popular sátira cómica se inspira en parodias sobre escenas comunes en este tipo de barrios y caricaturiza los estereotipos de sus residentes, quienes son retratados como individuos incompetentes y antisociales que viven en hogares disfuncionales y buscan evitar el trabajo. En resumen, se trata de una degradación de los valores centrales de la ascendente ética neoliberal basada en la "responsabilidad individual". El diseño conjunto de este estudio permite a los inquilinos que forman parte esta iniciativa expresar sus pensamientos y sentimientos sobre los "vínculos entre el lugar y la desventaja", a la vez que se confirma el poder de permanencia de la estigmatización territorial entre no-inquilinos. ${ }^{42}$

El artículo elaborado por Kallin y Slater, titulado Gentrifying Marginality on Edinburgh's Periphery ${ }^{43}$, se centra en la relación entre la degradación activa de áreas obreras deterioradas y su remodelación dirigida por y hacia intereses privados mediante políticas públicas destinadas a familias de clase media. Tras recopilar material de archivo y realizar entrevistas en profundidad a nuevos y antiguos residentes, los autores de esta colaboración se adentran en la concepción e implementación del

42 Este artículo confirma de forma indirecta el vínculo orgánico detectado por Morris (2013), entre la diseminación de la marginalidad urbana y la degradación espacial en el sector de la vivienda pública de Australia.

43 Kallin y Slater, 2014. 
reciente proyecto de "regeneración" que pretende convertir al barrio de Craigmillar, un enclave de pobreza extrema resultante de la desindustrialización ubicado en el extremo sudeste de la capital de Escocia, en un ejemplo de renacimiento urbano. Aquí se revela cómo funcionarios públicos elaboraron y vincularon el concepto de desprestigio territorial con este barrio para posteriormente justificar la demolición de sus dos vecindarios, ignorando completamente las necesidades colectivas de sus habitantes. Este estudio de caso representa la manipulación política multidimensional respecto a la estigmatización territorial, la cual proporciona un punto de partida simbólico y un objetivo práctico de gentrificación estatal y fomenta la censura de políticas alternativas de inversión social que podrían hacer frente a la pobreza y al deteriorado estado de las viviendas en un área determinada. ${ }^{44}$

Sakizlioğlu y Uitermark, en su artículo Comparing the Gentrification of Stigmatized Neighborhoods in Amsterdam and Istanbul ${ }^{45}$ brindan luces sobre las políticas simbólicas de desplazamiento urbano. Sin embargo, a pesar de las profundas diferencias a nivel de estructuras estatales, gamas políticas y organización urbana, los autores de esta investigación

44 Esta estrategia es fuertemente defendida en el supuesto urgente caso de la "zona urbana" norteamericana (remanentes del histórico gueto negro) propuesto por Mary Patillo (2008) en su provocativo trabajo Investing in Poor Black Neighborhoods "As $I s^{\prime \prime}$.

45 Sakizlioğlu y Uitermark, 2014. revelan dos sorprendentes semejanzas dentro del curso socio-político del proceso de gentrificación en estas dos ciudades globales ubicadas en Holanda y Turquía. En ambos lugares, mientras que los partidarios del mejoramiento barrial se embarcan en sutiles campañas de "divide y vencerás" a nivel cultural mediante el establecimiento de clasificaciones que dividen a la población local en categorías desconectadas o antagónicas, aquellos que apoyan iniciativas privadas utilizan el tiempo a su favor para desgastar y diferenciar aún más a sus oponentes. La combinación de estas dos estrategias previene, limita o simplemente anula efectivamente todo tipo de resistencia individual o colectiva ante desplazamientos y también actúa en casos donde el acceso a nuevas áreas vecinales ocurre sin la necesidad de recurrir a movilizaciones. La habilidad de personas y lugares de moverse de una clasificación a otra es una parte esencial de la transformación material del paisaje urbano.

Por último, Troels Schultz Larsen nos invita a recorrer un viaje histórico en su artículo titulado Copenhaguen's West End as a "Paradise Lost", donde analiza la producción política y usos del estigma territorial en esta metrópolis danesa. Este 
autor explora la trayectoria involutiva del barrio de Tåstrupgård, el cual pasó de ser un prestigioso espacio precursor de la alta modernidad hace medio siglo a un reducto deteriorado y dominado por luchas por espacio. La combinación de la teoría de campo de Bourdieu con observación etnográfica le permite a Larsen demostrar que la consolidación de la marginalidad avanzada y la correspondiente difusión de la estigmatización territorial en Copenhague son el resultado de decisiones de política derivadas de disputas a nivel burocrático. Estas contiendas han determinado la concentración de viviendas desposeídas y la denigración colectiva de sus barrios a través de la institucionalización de un mercado inmobiliario asimétrico y la dualidad de políticas urbanas que privilegian la propiedad privada a expensas de la atribución pública de viviendas. En este caso el Estado también emerge como una institución, esfera y actor esencial en el contexto de los choques materiales y simbólicos que producen, esparcen y validan el estigma espacial, al punto que el problema diario generado por

236 revista invi № 82 / Noviembre 2014 / Volumen № 29: 219-240 la precariedad urbana no puede ser comprendido -y menos aún resuelto- por medio de un limitado enfoque centrado en los barrios donde nace este problema. ${ }^{46}$

La estigmatización territorial no es una condición estática, un proceso neutral o un juego cultural inofensivo, sino una forma significativa y perjudicial de acción mediante la representación colectiva centrada en un lugar determinado. Al mostrar el modo en que las propiedades y mecanismos de operación de la estigmatización espacial se manifiestan en distintos tipos de escenarios urbanos y formaciones políticas, los colaboradores del número ofrecido por Environment and Planning A nos ayudan a progresar en nuestra comprensión sobre el papel de las estructuras simbólicas en la producción de desigualdad y marginalidad en la ciudad y, más aún, enriquecer nuestro dominio teórico respecto a las conexiones flexibles entre espacio social, espacio simbólico y espacio físico. ${ }^{47}$ Estos artículos nos entregan abundante material para elaborar una imagen más compleja y matizada sobre la estructura social,

46 Schultz Larsen (2014) también se involucra exitosamente en e creciente volumen de obras teóricas y empíricas escritas por académicos escandinavos, quienes han reflexionado y probado la aplicabilidad del concepto de estigmatización espacial elaborado por Wacquant en las sociedades nórdicas. Estas últimas están caracterizadas por sus reducidas desigualdades urbanas y por su el carácter socialmente inclusivo de sus ciudadanos (consultar Sernhede 2009; Delica, 2011; Jensen y Christensen, 2012; y Jensen et al. en la prensa escrita y en las citas referidas por estos autores)

47 Wacquant, 2014a. 
dinámicas e identidad de estos barrios desprestigiados de la metrópolis polarizadora, los cuales suscitan una renovada fascinación de parte de los medios de comunicación, así como preocupación a nivel político y atención académica a través de las fronteras nacionales. Asimismo, estas contribuciones son una respuesta directa al difundido retrato de estos espacios como calderas hirvientes de decadencia social, disipación moral y debilidad nacional. El objetivo de estos artículos es subrayar la necesidad urgente de medidas políticas enfocadas no sólo en la reducción de carencias materiales sino también en la disminución de la presión ejercida por la dominación simbólica en la metrópolis.

\section{Bibliografía}

ARTHURSON, Kathy; DARCY, Michael y ROGERS, DaIlas. Televised territorial stigma: how social housing tenants experience the fictional media representation of estates in Australia. [En línea]. Environment \& Planning A. 46(6): 1334-1350, 2014. ISSN 1472-3409. Disponible en: http://dx.doi. org/10.1068/a46136

AUGUST, Martine. Challenging the rhetoric of stigmatization: the benefits of concentrated poverty in Toronto's Regent Park. [En línea]. Environment \& Planning A. 46(6): 1317-1333, 2014. ISSN 14723409. Disponible en: http://dx.doi.org/10.1068/ a 45635

ARTíCULO: Estigmatización territorial en acción /

Loïc Wacquant, Tom Slater, Virgilio Borges Pereira
AUYERO, Javier. 'This is a lot like the Bronx, isn't it?' Lived experiences of marginality in an Argentine slum. [En línea]. International Journal of Urban and Regional Research. 23(1): 45-69, 1999. ISSN 1468-2427. Disponible en: http://dx.doi. org/10.1111/1468-2427.00178

BECKETT, Katherine y HERBERT, Steve. Banished: the new social control in urban America. New York, Oxford University Press. 2011.

BIRDSALL-JONES, Christina. The Bronx in Australia The metaphoric stigmatization of public housing tenants in Australian towns and cities. [En línea]. Journal of Urban History. 39(2): 315-330, 2013. ISSN 1552-6771. Disponible en: http://dx.doi. org/10.1177/0096144212465265

BOURDIEU, Pierre. Language and symbolic power. Cambridge, UK, Polity Press. 1991.

CHICAGO Tribune (Staff of the). The American millstone: an examination of the nation's permanent underclass. Chicago, Contemporary Books. 1986.

CLEAR, Todd R. Imprisoning communities: how mass incarceration makes disadvantaged neighborhoods worse. New York, Oxford University Press. 2007.

DELICA, Kristian Nagel. Sociologisk refleksivitet og feltanalytisk anvendelse af etnografi: om Loîc Wacquants blik på urban marginalisering. [En línea]. Dansk Sociologi. 22(1): 27-45, 2011. Disponible en: http://ej.lib.cbs.dk/index.php/ dansksociologi/article/view/3474

revista invi № 82 / Noviembre 2014 / Volumen № 29: 219-240 237 
ENGELS, Friedrich. The condition of the working class in England. Oxford, Oxford University Press. [1845] 1993.

GOFFMAN, Erving. Stigma: notes on the management of spoiled identity. New York, Simon \& Schuster. 1963.

HUSSEY, Andrew. The French Intifada: the long war between France and its Arabs. New York, Faber \& Faber. 2014.

IBRAHIMOVIĆ, Zlatan y LAGERCRANTZ, David. I am Zlatan: My story on and off the field. New York, Random House. 2014.

JENCKS, Christopher, ed. and PETERSON, Paul E., ed. The urban underclass. Washington, DC, The Brookings Institution. 1991.

JENSEN, Sune Qvotrup y CHRISTENSEN, Ann-Dorte. Territorial stigmatization and local belonging: a study of the Danish neighbourhood Aalborg East. [En línea]. City. 16(1-2): 74-92, 2012. ISSN 1470-3629. Disponible en: http://dx.doi.org/10.10 80/13604813.2012.663556

JENSEN, Sune Qvotrup; PRIEUR, Annick y SKJØTTLARSEN, Jakob. Living with stigma: spatial and social divisions in a Danish city. International Journal of Urban and Regional Research. ISSN 1468-2427. Número especial "Taking Bourdieu to Town." En prensa.

KALIFA, Dominique. Les bas-fonds. Histoire d'un imaginaire. Paris, Seuil. 2012
KALLIN, Hamish y SLATER, Tom. Activating territorial stigma: gentrifying marginality on Edinburgh's periphery. [En línea]. Environment \& Planning A. 46(6): 1351-1368, 2014. ISSN 1472-3409.. Disponible en: http://dx.doi.org/10.1068/a45634

KATZ, Michael B. The undeserving poor: America's enduring confrontation with poverty. New York, Oxford University Press. 2013.

KIRKNESS, Paul. The cités strike back: restive responses to territorial taint in the French Banlieues. [En línea]. Environment \& Planning A. 1281-1296. Disponible en: http://dx.doi.org/10.1068/a45636

KOVEN, Seth. Slumming: sexual and social politics in Victorian London. Princeton, Princeton University Press. 2006.

MAYHEW, Robert. London labour and the London poor. Editado por Robert Douglas-Fairhurst. Oxford, Oxford University Press. [1851] 2012.

MERTON, Robert King. Sociological ambivalence and other essays. New York, The Free Press. 1976.

MORRIS, Alan. Public housing in Australia: A case of advanced urban marginality? [En línea]. The Economic \& Labour Relations Review. 24(1): 8096, 2013. ISSN 1838-267. Disponible en: http:// dx.doi.org/10.1177/1035304612474215

MUCCHIELLI, Laurent, ed. La frénésie sécuritaire. Retour à l'ordre et nouveau contrôle social. Paris, La Découverte. 2008a.

PATTILLO, Mary. Investing in poor black neighborhoods 'As Is'. En: Turner, Margery; POPKIN, 
Susan y RAWLINGS, Lynette. Public housing and the legacy of segregation. Washington, DC, Urban Institute Press. 2008. p. 31-46

PECK, Jamie y THEODORE, Nik. Carceral Chicago: making the ex-offender employability crisis. [En línea]. International Journal of Urban and Regional Research. 32(2): 251-281, 2008. ISSN 1468-2427. Disponible en: http://dx.doi. org/10.1111/j.1468-2427.2008.00785.x

PEREIRA, Virgílio Borges y QUEIRÓS, João Pedro Luís de. "It's not a bairro, is it?": subsistence sociability and focused avoidance in a public housing estate. Environment \& Planning A. 46(6): 1297-1316, 2014. ISSN 1472-3409. Disponible en: http:// dx.doi.org/10.1068/a46300

PEREIRA, Virgílio Borges. Classes e culturas de classe das famílias portuenses: classes sociais e "modalidades de estilização da vida" na cidade do Porto. Porto, Afrontamento. 2005.

PRED, Allan. Even in Sweden: racisms, racialized spaces, and the popular geographical imagination. Berkeley, University of California Press. 2000.

SAKIZLIOĞLU, Bahar y UITERMARK, Justus. The symbolic politics of displacement: comparing the gentrification of stigmatized neighborhoods in Amsterdam and Istanbul. Environment \& Planning A. 46(6): 1369-1385, 2014. ISSN 1472-3409. Disponible en: http://dx.doi.org/10.1068/a45638

SCHULTZ LARSEN, Troels. Copenhagen's West End a 'Paradise Lost': the political production and uses of territorial stigma in Denmark. Environment $\mathcal{E}$
Planning A. 46(6): 1386-1402, 2014. ISSN 14723409. Disponible en: http://dx.doi.org/10.1068/ a45640

SERNHEDE, Ove. Territorial Stigmatisering: unges uformelle læring og skolen i det postindustrielle samfund. Social Kritik. 21(118): 5-23, 2009.

SLATER, Tom y ANDERSON, Ntsiki. The reputational ghetto: territorial stigmatisation in St Paul's, Bristol. [En línea]. Transactions of the Institute of British Geographers. 37(4): 530-546, 2012. ISSN 1475-5661. Disponible en: http://dx.doi. org/10.1111/j.1475-5661.2011.00490.x

SLATER, Tom et al. An international bibliography on territorial stigmatization. [En línea]. 2013. Disponible en: http://www.srdg.co.uk/homes/tslater/ territorialstigmabiblio.pdf.

SLATER, Tom. The eviction of critical perspectives from gentrification research. [En línea]. International Journal of Urban and Regional Research. 30(4): 737757, 2006. ISSN 1468-2427. Disponible en: http:// dx.doi.org/10.1111/j.1468-2427.2006.00689.x

- The myth of broken Britain: welfare reform and the production of ignorance. Antipode. En prensa. 2014.

TRUONG, Fabien. Des capuches et des hommes. Trajectoires de "jeunes de banlieue". Paris, BuchetChastel. 2013.

WACQUANT, Loïc. Territorial stigmatization in the age of advanced marginality. [En línea]. Thesis Eleven. 91(1): 66-77, November 2007. 
ISSN 1461-7455. Disponible en: http://dx.doi. org/10.1177/0725513607082003

- Urban outcasts: a comparative sociology of advanced marginality. Cambridge, UK, Polity Press. 2008.

- The body, the ghetto and the penal state. [En línea]. Qualitative Sociology. 32(1): 101-129, March 2009a. ISSN 1573-7837. Disponible en: http:// dx.doi.org/10.1007/s11133-008-9112-2

- Punishing the poor: the neoliberal government of social insecurity. Durham and London, Duke University Press. 2009b.

- Designing urban seclusion in the 21st century. Perspecta: The Yale Architectural Journal. 43: 165178,2010

A janus-faced institution of ethnoracial closure: a sociological specification of the ghetto. En: HUTCHISON, Ray, ed. y HAYNES, Bruce, ed. The ghetto: contemporary global issues and controversies. Boulder, Westview. 2011a. p. 1-31

- Territorial stigmatization revisited: symbols, state, and space. Keynote address to the International Conference on Territorial Stigmatization and Advanced Marginality, University of Porto, Porto, Portugal, June 22. 2011b.

- Marginality, ethnicity and penality in the neoliberal city: an analytic cartography. Ethnic $\&$ Racial Studies Review, Symposium: En prensa. 2014a.
Revisiting territories of relegation: class, ethnicity and the state in the making of marginality. Urban Studies Journal, Symposium: En prensa. 2014b. 